\title{
The utility of gingival crevicular fluid matrix metalloproteinase-8 provides site-specific diagnostic value for periodontal grading
}

\author{
MAŁGORZATA NĘDZI-GÓRA ${ }^{1}$, RENATA GÓRSKA², BARTŁOMIEJ GÓRSKI ${ }^{2,3}$ \\ ${ }^{1}$ Department of Oral Hygiene, Medical University of Warsaw, Warsaw, Poland \\ ${ }^{2}$ Department of Periodontology and Oral Mucosa Diseases, Medical University of Warsaw, Warsaw, Poland \\ ${ }^{3}$ The Chair and Department of Periodontology, Medical University of Lublin, Lublin, Poland
}

\begin{abstract}
Introduction: Matrix metalloproteinase-8 (MMP-8), and its active form aMMP-8, was identified as a potential biomarker of periodontal tissue destruction. It is present at different concentrations in various oral fluids.

Material and methods: Gingival crevicular fluid (GCF) samples were collected from periodontal pockets $\geq 6 \mathrm{~mm}$ of 24 untreated patients using paper points and clinical parameters were recorded. 12 subjects were diagnosed with periodontitis stage III grade B, and 12 others with periodontitis stage III grade $C$. After thorough preparations, samples were collected following manufacturers' instructions and analyzed using a commercially available test system for aMMP-8 evaluation (aMMP-8 Test) and Periotron 8000 together with Quantikine kits for assessment of total MMP-8 concentration (controls). Microbiological evaluation of the same pockets was carried out using real-time polymerase chain reaction.

Results: Concentrations of both total MMP-8 and aMMP-8 in GCF were higher in the case of periodontitis grade $C$, compared to periodontitis grade $B$, but reached statistical significance only in the case of total MMP-8 $(77.17 \mathrm{ng} / \mathrm{ml}$ and $18.73 \mathrm{ng} / \mathrm{ml}$ respectively; $p=0.0104)$. Positive correlations were found between total MMP-8 and aMMP-8 levels and the prevalence of Fusobacterium nucleatum, mean probing pocket depth of all pockets, $\%$ of pockets $\geq 6 \mathrm{~mm}$, as well as probing pocket depth of pocket from which GCF samples were collected.

Conclusions: GCF concentration levels of both total MMP-8 and aMMP-8 correlated with severity of periodontal destruction, whereas total MMP-8 appeared to be a preferable method for differentiation of periodontal grading. However, the aMMP-8 Test was easier and more convenient to handle.
\end{abstract}

Key words: periodontal disease, biomarker, metalloproteinases, gingival crevicular fluid.

(Cent Eur J Immunol 2021; 46 (2): 236-243)

\section{Introduction}

Matrix metalloproteinase-8 (MMP-8), also named collagenase- 2 or neutrophil collagenase, is an endoprotease which governs degradation of both periodontal soft and hard tissues that are mostly built of type I collagen. This process might take place during physiological remodeling, as well as under irreversible pathologic breakdown. MMP8 is released from neutrophils through degranulation. Polymorphonuclear leukocytes provoked in vitro secretion of MMP-8 from intracellular granules within a few seconds after a stimulus [1]. This reaction may be stipulated by periodontal microbiota, microbial virulence factors and a plethora of proinflammatory mediators (e.g. interleukin $1 \beta$, tumor necrosis factor $\alpha$ ) [2]. Constant interplay between MMP-8 activity and its natural regulators, such as tissue inhibitors of MMP (TIMPs) and $\alpha 2$-macroglobulin, plays a role in regulation of periodontal tissue homeostasis.

According to a recent systematic review, an elevated concentration of latent or total MMP-8, and particularly its active/activated form (aMMP-8, Mr $65 \mathrm{kDa}$ ), was associated with progressive periodontal and peri-implant inflammatory diseases, and also with more advanced stages of periodontitis compared with healthy and intact periodontium $[3,4]$. The MMP-8 activation in oral fluids reflected conversion of gingivitis to periodontitis, and peri-mucositis to peri-implantitis at site and patient levels of aMMP-8 [3-6]. Upregulation in oral fluids preceded progression of periodontitis/peri-implantitis and contributed to a detectable increase in attachment loss [5-7]. Moreover, concentration of MMP- 8 declined after effective periodontal therapy [8]. Therefore MMP-8 seems to be a reliable marker

Correspondence: Bartłomiej Górski, PhD, Department of Periodontology and Oral Mucosa Diseases, Medical University of Warsaw, Stanisława Binieckiego 6 St., 02-097 Warsaw, Poland, e-mail: bartek_g3@tlen.pl Submitted: 6.05.2020; Accepted: 11.08.2020 
that may facilitate risk assessment of periodontitis occurrence and dynamics of its course. The level of MMP- 8 can be assessed in different oral fluids, such as saliva, mouth rinse, gingival crevicular fluid (GCF) and peri-implantitis sulcular fluid (PISF). As saliva or mouth rinse assay can be beneficial for screening objectives, an analysis of biochemical components of GCF reflects the degree of collagen catabolism and conditions within periodontal tissues [9]. It was estimated that $90 \%$ to $95 \%$ of collagenolytic activity in GCF originate from MMP-8 [10]. The standard gold laboratory-based test for total MMP-8 immunoactivities in GCF is enzyme-linked immunosorbent assay (ELISA). However, several other technologies, which involved ready-made diagnostic kits and chair-side analysis of raised aMMP-8, not only in GCF and PISF, but also in saliva and mouth rinse, were proposed $[11,12]$. Some authors recommended analyzing associations of periodontal microbiota in oral fluids with concentration of MMP-8 with a view to enhancing diagnostic precision [13, 14].

Keeping that in mind, one aim of this study was to determine whether total MMP-8 GCF levels and aMMP-8 GCF concentrations measured by two different methods may distinguish subjects with two different periodontal diagnoses. Another aim was to detect associations between evaluated concentrations of collagenase- 2 and clinical parameters, as well as prevalence of selected periodontal microbiota.

\section{Material and methods}

This study received a positive approval by the institutional review board (KB/171/2009) and was carried out at the Department of Periodontology and Oral Mucosa Diseases of Medical University of Warsaw. All clinical procedures were performed in accordance with the Helsinki Declaration of 1975, as revised in Tokyo in 2004. Written and signed informed consent forms were obtained from every patient.

The following inclusion criteria were adopted: 1) diagnosed periodontitis stage III grade B or grade C, 2) presence of at least one periodontal pocket $\geq 6 \mathrm{~mm}$ deep, 3) no coexisting systemic conditions that may affect the course of periodontal disease, 4) no chronic intake of drugs that can modify the course of periodontal disease (antibiotics, steroids, anti-inflammatory drugs, immunosuppressants, antiepileptic drugs and calcium channel blockers), 5) no pregnant/lactating women, 6) no smoking, 7) no professional teeth cleaning within 3 months before the examination, 8) no topical use of products based on chlorhexidine within 3 months preceding the examination.

The study consisted of a clinical and laboratory part. Periodontal examination was performed by a calibrated examiner who used a graded periodontal probe (UNC probe $15 \mathrm{~mm}$, Hu-Friedy, Chicago, USA) and evaluated: 1) the number of teeth present in the oral cavity, 2) full-mouth plaque index (FMPI) according to O'Leary et al. [15] on four tooth surfaces as the number of surfaces with plaque divided by the number of all examined surfaces; 3 ) full mouth bleeding on probing index (FMBOP) according to Ainamo and Bay [16] at six points for each tooth (i.e. distobuccal, buccal, mesiobuccal, distolingual, lingual, mesiolingual) by dividing the number of bleeding points by the number of all probed points; 4) probing pocket depth (PPD) at six points of each tooth as the distance from the gingival margin to the bottom of the pocket; 5) clinical attachment level (CAL) at six points of each tooth as the distance from the cemento-enamel junction (CEJ) to the bottom of the pocket. On account of clinical examination and evaluation of panoramic and periapical X-rays, diagnosis of periodontitis was made [2]. Periodontitis stage III was recognized when: 1) interdental CAL $\geq 5 \mathrm{~mm}$, 2) radiographic bone loss extended to the mid-third of the tooth root and beyond, 3) tooth loss due to periodontitis was $\leq 4$ teeth, 4) PPD $\geq 6 \mathrm{~mm}, 5$ ) vertical bone loss $\geq 3 \mathrm{~mm}$. Periodontitis grades were assessed indirectly on dental radiograms as percentage of root length divided by the age of the subject (\% bone loss/age). Periodontitis grade B was identified when this value varied from 0.25 to 1.0 , whereas periodontitis grade $\mathrm{C}$ was identified when it was $>1.0$.

In order to collect a sample of GCF the deepest pocket $\geq 6 \mathrm{~mm}$ was selected for each patient. Sample collection was carried out on the day following periodontal examination. All patients were asked to refrain from eating, drinking, and tooth brushing on the day prior to sample collection. Gingival plaque was removed from the tooth with a swab, then the examined area was dried and isolated from the excess saliva. Next, with sterile tweezers one paper point (Paperstrip, OraFlow Inc., USA) was inserted into the pocket for 30 seconds and subsequently placed into a device assigned to evaluate the volume of the collected GCF (Periotron 8000, OraFlow Inc., USA). In the case of the paper point being contaminated with blood or saliva, the procedure was rerun. Periotron was calibrated using standard volumes of human saliva $(0.25 \mu \mathrm{l}, 0.75 \mu \mathrm{l}, 1.0 \mu \mathrm{l}, 1.25 \mu \mathrm{l})$ measured with a micropipette. Paper points were put into tubes containing $500 \mu \mathrm{l}$ of phosphate-buffered saline, frozen to the temperature $-20^{\circ} \mathrm{C}$ and stored until biochemical analysis. GCF concentrations of MMP- 8 were assessed by an enzyme-linked immunosorbent assay (ELISA) using commercially available Quantikine kits (R\&D Systems, MN, USA) for quantitative analysis of total MMP-8 (test sensitivity $0.06 \mathrm{ng} / \mathrm{ml}$, assay range $0.2-10 \mathrm{ng} / \mathrm{ml}$ ).

A commercially available test system (aMMP-8 Test, Advanced Dental Diagnostics, Malden, Netherlands) was used following the manufacturer's instructions. In brief, paper strips were placed in designated periodontal pockets for 30 seconds, and then placed into labeled tubes, which were sent to the laboratory. In the designated laboratory the Sandwich ELISA method was used to measure the concentration of aMMP-8 in the sample (test sensitivity $94.4 \%$, test specificity $100 \%$ ). Antibodies used in the test identified specifically activated forms of MMP-8. 
Table 1. Clinical characteristics of groups (mean with $95 \%$ confidence interval and standard deviation).

\begin{tabular}{lccc}
\hline Variable & $\begin{array}{c}\text { Periodontitis grade B } \\
(\boldsymbol{n}=\mathbf{1 2})\end{array}$ & $\begin{array}{c}\text { Periodontitis grade C } \\
(\boldsymbol{n}=\mathbf{1 2})\end{array}$ & $\boldsymbol{p}$ (Student $\boldsymbol{t}$-test) \\
\hline Number of teeth & $25.12[22.84-27.40] \pm 1.10$ & $24.66[21.27-28.05] \pm 1.53$ & 0.4069 \\
\hline $\begin{array}{l}\text { Mean probing pockets } \\
\text { depth (PPD) }(\mathrm{mm})\end{array}$ & $2.75[2.21-3.28] \pm 0.24$ & $3.23[2.42-4.04] \pm 0.36$ & $0.0009^{*}$ \\
\hline $\begin{array}{l}\% \text { of pockets with PPD } \\
\geq 6 \text { mm }(\%)\end{array}$ & $6.91[3.66-10.17] \pm 1.47$ & $14.41[5.09-23.74] \pm 4.23$ & $<0.0001^{*}$ \\
\hline $\begin{array}{l}\text { Full mouth plaque score } \\
\text { (FMPI) (\%) }\end{array}$ & $50.25[37.22-63.27] \pm 5.91$ & $47.58[31.08-64.08] \pm 7.49$ & 0.3429 \\
\hline $\begin{array}{l}\text { Full mouth bleeding on } \\
\text { probing (FMBOP) }(\%)\end{array}$ & $51.58[39.71-63.44] \pm 5.39$ & $51.83[32.81-70.85] \pm 8.64$ & 0.9330 \\
\hline MMP-8 (ng/ml) & $28.73[17.92-39.54] \pm 4.91$ & $77.17[43.07-111.26] \pm 15.49$ & $<0.0001^{*}$ \\
\hline aMMP-8 (ng/ml) & $24.00[14.88-33.12] \pm 15.80$ & $49.09[12.52-85.66] \pm 54.44$ & 0.1395 \\
\hline *Statistically significant difference at $p<0.05$ & &
\end{tabular}

*Statistically significant difference at $p<0.05$

Subsequently, microbiological analysis of the same periodontal pocket was performed using a commercially available PET diagnostic kit (MIP Pharma, Icking, Germany) in accordance with the instructions provided by the manufacturer. Briefly, the paper point was inserted into the pocket for 20 seconds, then packed into a designated test tube and sent to the manufacturer's laboratory. The total number of bacteria and the accurate number of nine periopathogens in the sample (Prevotella intermedia, Peptostreptococcus micros, Fusobacterium nucleatum, Eubacterium nodatum, Porphyromonas gingivalis, Treponema denticola, Tannerella forsythia, Aggregatibacter actinomycetemcomitans, Capnocytophaga gingivalis) were assessed with real-time polymerase chain reaction (real-time PCR).

Statistical analysis was performed with Statistica v. 13 (TIBCO Software Inc., Palo Alto, USA). Data were presented as mean \pm standard deviation (SD) and $95 \%$ confidence intervals. The unequal variance Student $t$-test was used for comparisons of two independent groups for continuous variables. The normality of the data distribution was verified by the Shapiro-Wilk test and the equality of variances was assessed used the Levene test. For all study parameters the data distribution did not differ significantly from the normal distribution, and the variances of the compared variants were homogeneous. Relationships between concentrations of MMP-8 and evaluated clinical and microbiological parameters were assessed using Spearman rank correlation $(R)$. The distributions and prevalence of selected periodontal microbes between study groups were analyzed by chi-squared test. $P$ values of less than 0.05 $(p<0.05)$ were regarded as statistically significant.

\section{Results}

The study included 24 patients: 12 individuals ( 7 females and 5 males with an average age of 48.4 years) with periodontitis grade $B$ and 12 subjects ( 7 females and 5 males with an average age of 40.3 years) with periodontitis grade C. The groups' characteristics are depicted in Table 1. Individuals with periodontitis grade $\mathrm{C}$ had significantly more deep periodontal pockets. Concentrations of both total MMP-8 and aMMP-8 in GCF were higher in the case of periodontitis grade $\mathrm{C}$ compared to periodontitis grade $\mathrm{B}$. However, a statistically significant difference was observed only for total MMP-8 $(77.17 \mathrm{ng} / \mathrm{ml}$ and $28.73 \mathrm{ng} / \mathrm{ml}$, respectively, $p=0.0104$ ) evaluated with ELISA technique.

No significant differences between grade $\mathrm{B}$ and grade $\mathrm{C}$ were observed in terms of distribution and the number of evaluated periopathogens with the exception of T. forsythia (Table 2). T. forsythia was more often found in patients diagnosed with grade $\mathrm{C}$ periodontitis $(p=0.0372$ ).

Positive correlations were found between MMP- 8 and aMMP-8 concentration and the prevalence of $F$. nucleatum in the sample, mean PPD of all pockets, $\%$ of pockets deeper than $6 \mathrm{~mm}$, as well as PPD of pocket from which the GCF sample was collected (Table 3). Moreover, a statistically significant correlation between aMMP-8 level and presence of dental plaque on the surface of the evaluated tooth was found in the test group.

\section{Discussion}

Case definition of periodontitis is currently based on a combination of periodontitis staging and grading [17]. As staging depends on the severity and extent of periodontitis, grading outlines the periodontitis progression rate and relative risk of future tooth loss. Periopathogens in the subgingival biofilm are considered a primary etiological factor of periodontitis, but the occurrence and progression of the disease are determined by the genetically programmed immune response of the host [18]. Among a number of bioactive agents that have been suggested as potential biomarkers of periodontitis, the value of MMP-8 was highlighted [3]. In this context, we hypothesized that 
Table 2. Microbiological characteristics of groups (number and percentage of individuals with detected microbiota)

\begin{tabular}{|c|c|c|c|c|c|c|}
\hline Periopathogen & $\begin{array}{c}\text { Periodontitis } \\
\text { grade }\end{array}$ & $\begin{array}{c}\text { Under detection } \\
\text { limit }\left(<10^{3}\right)\end{array}$ & $10^{3}$ & $10^{4}$ & $>10^{5}$ & $p\left(\chi^{2}\right.$ test $)$ \\
\hline \multirow[t]{2}{*}{ Porphyromonas gingivalis } & $\mathrm{C}$ & $8(67)$ & $2(17)$ & $2(17)$ & $0(0)$ & 0.3826 \\
\hline & B & $9(75)$ & $2(17)$ & $0(0)$ & $1(8)$ & \\
\hline \multirow[t]{2}{*}{ Treponema denticola } & $\mathrm{C}$ & $4(33)$ & $5(42)$ & $3(25)$ & $0(0)$ & 0.0655 \\
\hline & B & $9(75)$ & $2(17)$ & $0(0)$ & $1(8)$ & \\
\hline \multirow[t]{2}{*}{ Tannerella forsythia } & $\mathrm{C}$ & $6(50)$ & $2(17)$ & $4(33)$ & $0(0)$ & $0.0372 *$ \\
\hline & B & $11(92)$ & $0(0)$ & $0(0)$ & $1(8)$ & \\
\hline \multirow[t]{2}{*}{ Prevotella intermedia } & $\mathrm{C}$ & $10(83)$ & $1(8)$ & $1(8)$ & $0(0)$ & 0.6694 \\
\hline & B & $8(67)$ & $1(8)$ & $2(17)$ & $1(8)$ & \\
\hline \multirow[t]{2}{*}{ Peptostreptococcus micros } & $\mathrm{C}$ & $7(58)$ & $4(33)$ & $1(8)$ & $0(0)$ & 0.8213 \\
\hline & B & $9(75)$ & $2(17)$ & $1(8)$ & $0(0)$ & \\
\hline \multirow[t]{2}{*}{ Fusobacterium nucleatum } & $\mathrm{C}$ & $4(33)$ & $5(42)$ & $3(25)$ & $0(0)$ & 0.4180 \\
\hline & B & $8(67)$ & $3(25)$ & $1(8)$ & $0(0)$ & \\
\hline \multirow[t]{2}{*}{ Eubacterium nodatum } & $\mathrm{C}$ & $11(92)$ & $1(8)$ & $0(0)$ & $0(0)$ & 1 \\
\hline & B & $11(92)$ & $1(8)$ & $0(0)$ & $0(0)$ & \\
\hline \multirow[t]{2}{*}{ Capnocytophaga gingivalis } & $\mathrm{C}$ & $5(42)$ & $5(42)$ & $2(17)$ & $0(0)$ & 0.6108 \\
\hline & B & $7(58)$ & $2(17)$ & $3(25)$ & $0(0)$ & \\
\hline \multirow{2}{*}{$\begin{array}{l}\text { Aggregatibacter } \\
\text { actinomycetemcomitans }\end{array}$} & $\mathrm{C}$ & $11(92)$ & $0(0)$ & $1(8)$ & $0(0)$ & 0.7908 \\
\hline & B & $12(100)$ & $0(0)$ & $0(0)$ & $0(0)$ & \\
\hline
\end{tabular}

* Statistically significant difference at $p<0.05$

MMP-8 and aMMP-8 would be associated with dynamic periodontal disease progression rate, periodontal parameters and periodontal microbiota. In order to guarantee that outcomes are not influenced by general health, medication or habits, only generally healthy and non-smoking patients were included in the study. By and large, both total MMP-8 and active MMP-8 levels in GCF were higher in subjects with clinical diagnosis of periodontitis grade $\mathrm{C}$. However, only in the case of total MMP- 8 concentration was the observed relationship statistically significant. Moreover, the levels of measured metalloproteinase correlated positively with mean probing pocket depth of all pockets and $\%$ of pockets deeper than $6 \mathrm{~mm}$, which reflected the status of general periodontal condition. Lack of statistically significant differences in FMPI and FMBOP between grade $\mathrm{B}$ and grade $C$ patients points to the fact that evaluated biomarkers may represent valid indicators in terms of differential diagnosis. On the other hand, the levels of both total and active MMP-8 showed a positive correlation with the prevalence of $F$. nucleatum, as well as with PPD of the periodontal pocket from which GCF was collected, thus pinpointing active and ongoing inflammatory processes within the specified area. All things considered, the diagnostic process with the commercially available aMMP-8 Test was easier and more convenient to handle; hence it did not require organizational structure (Periotron) or additional steps (calibration of Periotron, freezing of GCF samples).
In the present study MMP-8 concentration was evaluated in GCF, which provided site-specific and exact data regarding the measured periodontal pocket. Due to the limited availability of GCF, longer time is required to collect the sample. Moreover, the paper point might easily get contaminated with blood, saliva or supragingival plaque [19]. By the same token, collection of saliva or mouth rinse is simpler and less time-consuming; thus many researchers suggested using them as specimens. Saliva analysis may be useful for screening purposes, but is unable to give site-specific information [20]. The majority of studies suggested high sensitivity of saliva for discerning changes in MMP-8 levels in patients with periodontal disease relative to healthy controls $[11,22,23]$. For example, the study by Johnson et al. [23] demonstrated 4.1 times higher concentration of MMP-8 in saliva in periodontally diseased patients compared with periodontally healthy subjects. Two recent meta-analyses concluded that salivary MMP-8 levels were significantly higher in periodontitis patients compared with healthy controls $[12,24]$. On the other hand, the assessment of MMP- 8 concentration in GCF was claimed to be the most useful and effective for the diagnosis of periodontitis in systematically healthy subjects, irrespective of smoking condition [25]. All in all, for the purpose of periodontal disease progression assessment, GCF aMMP-8 displayed good sensitivity and excellent specificity [25-29]. 
Table 3. Correlations between the number of measured concentrations of MMP-8 in gingival crevicular fluid and evaluated clinical and microbiological parameters $(n=24)$ - Spearman rank correlation test

\begin{tabular}{lcc}
\hline & $\begin{array}{c}\text { MMP-8 } \\
(\mathbf{n g} / \boldsymbol{\mu l})\end{array}$ & $\begin{array}{c}\text { aMMP-8 } \\
\text { ADD }(\mathbf{n g} / \mathbf{m l})\end{array}$ \\
\hline Total number of periopathogens & -0.108 & -0.0269 \\
\cline { 2 - 3 } & $p=0.615$ & $p=0.899$ \\
\hline Porphyromonas gingivalis & -0.1721 & 0.0464 \\
\cline { 2 - 3 } & $p=0.613$ & $p=0.892$ \\
\hline Treponema denticola & -0.1636 & -0.0291 \\
\hline Tannerella forsythia & $p=0.560$ & $p=0.915$ \\
\hline Prevotella intermedia & 0.2956 & 0.3291 \\
\cline { 2 - 3 } & $p=0.351$ & $p=0.272$ \\
\hline Peptostreptococcus micros & -0.1994 & -0.0695 \\
\cline { 2 - 3 } & $p=0.581$ & $p=0.849$ \\
\hline Fusobacterium nucleatum & -0.1171 & 0.0244 \\
\hline Eubacterium nodatum & $p=0.644$ & $p=0.923$ \\
\hline Capnocytophaga gingivalis & 0.8487 & 0.8954 \\
\hline aggregatibacter & $p=0.000 *$ & $p=0.000 *$ \\
\hline Funycetemcomitans & -0.7636 & -0.3947 \\
\cline { 2 - 3 } & $p=0.236$ & $p=0.511$ \\
\hline & -0.1143 & -0.2043 \\
\hline & - & $p=0.388$ \\
\hline
\end{tabular}

\begin{tabular}{|c|c|c|}
\hline \multirow{2}{*}{$\begin{array}{l}\text { Full mouth plaque score (FMPI) } \\
(\%)\end{array}$} & 0.1607 & -0.3259 \\
\hline & $p=0.453$ & $p=0.112$ \\
\hline \multirow{2}{*}{$\begin{array}{l}\text { Full mouth bleeding on probing } \\
\text { (FMBOP) }(\%)\end{array}$} & 0.1003 & 0.2681 \\
\hline & $p=0.641$ & $p=0.195$ \\
\hline \multirow{2}{*}{$\begin{array}{l}\text { Mean probing pockets depth } \\
\text { (PPD) }\end{array}$} & 0.5112 & 0.5729 \\
\hline & $p=0.011^{*}$ & $p=0.003^{*}$ \\
\hline \multirow{2}{*}{$\begin{array}{l}\% \text { of pockets with } \mathrm{PPD} \geq 6 \mathrm{~mm} \\
(\%)\end{array}$} & 0.4871 & 0.4331 \\
\hline & $p=0.016^{*}$ & $p=0.031^{*}$ \\
\hline $\begin{array}{l}\text { Presence of plaque on evaluated } \\
\text { tooth }\end{array}$ & $\begin{array}{c}0.1341 \\
p=0.523\end{array}$ & $\begin{array}{c}0.4527 \\
p=0.023 *\end{array}$ \\
\hline $\begin{array}{l}\text { Presence of bleeding from } \\
\text { evaluated pocket }\end{array}$ & $\begin{array}{l}-0.1691 \\
p=0.419\end{array}$ & $\begin{array}{c}0.1838 \\
p=0.379\end{array}$ \\
\hline PPD of evaluated pocket & $\begin{array}{c}0.5138 \\
p=0.009 *\end{array}$ & $\begin{array}{c}0.5892 \\
p=0.002 *\end{array}$ \\
\hline
\end{tabular}

*Statistically significant difference at $p<0.05$

In a number of previous reports MMP-8 concentrations were investigated with ELISA or immunofluorometric assay (IFMA) techniques. Whereas ELISA senses all forms of MMP-8 (latent/total), IFMA recognizes activated neutrophil and fibroblast-type isoforms of aMMP-8 in a selective manner [14]. A recent systematic review stated that ELISA appeared to be the most suitable method [3]. Nevertheless, IFMA technique presented better results than ELISA and allowed periodontitis subjects to be selected from controls more strongly by salivary MMP-8 detection [19, 20, 30]. The MMP-8/TIMP-1 ratio separated periodontitis and control groups even in smoker subjects. The observed differences might be due to divergences and sensitivities of antibodies used in ELISA and IFMA [20]. In a study of Leppilahti et al. [30] MMP-8 level differentiated the study group with a strong inflammatory burden and was associated with higher MMP-8/TIMP-1 levels and elastase activity. Outcomes of IFMA were similar to DentoELISA but not to commercial Amersham ELISA, which utilize different antibodies. Moreover, positive correlations were reported between aMMP-8 GCF and PPD measured by IFMA, as well as between aMMP-8 and CAL evaluated by IFMA and DentoELISA in patients diagnosed with chronic periodontitis [31]. Salminen et al. [19] reported that high salivary MMP-8 level as measured by IFMA was associated with deepened periodontal pockets, bleeding on probing, and alveolar bone loss. On the other hand, total MMP-8 concentrations evaluated with the Amersham ELISA test showed no correlations with periodontal parameters $[13,14,22]$. Our presented data differ to some extent from the results of the abovementioned studies, since we noted positive correlations between total metalloproteinase levels and mean probing pocket depth, as well as $\%$ of pockets $\geq 6 \mathrm{~mm}$, but not with bleeding indices.

There is a pressing need for utilization of noninvasive screening and readily accessible diagnostic tools in clinical settings, such as point-of-care (PoC) chair side analysis to measure MMP-8/aMMP-8 levels in oral fluids to obtain prompt results. Such tests, which use lateral flow immunoassays with the detection limit of $20 \mathrm{ng} / \mathrm{ml}$, are already commercially available. The appearance of two lines on labeled strips indicates a positive result ( $>20 \mathrm{ng}$ aMMP- 8 per $\mathrm{ml}$ ) in the PerioSafe mouth-rinse test and the ImplantSafe strip test, which in turn signals elevated risk for formerly existing or developing periodontitis or peri-implantitis [2, 32, 33]. Both tests can be used for quantitative analysis with a designated reader (ORALyzer). Through evaluation of aMMP-8 in saliva PerioMarker was positive in $87 \%$ of chronic periodontitis subjects and in $40 \%$ of controls, which corresponded to sensitivity of $87 \%$ and specificity of $60 \%$ [34]. PoC tests were effective in detecting subclinical periodontitis/pre-periodontitis in adolescents, thus reducing the risk of their undertreatment [35]. Very recently aMMP- 8 measurements by an aMMP-8 PoC mouth rinse test with a cut-off of $20 \mathrm{ng} / \mathrm{ml}$ were used to evaluate periodontal grading. Consequently, it allowed patients with slow rate of periodontitis progression (grade A) to be distinguished from subjects with a moderate rate of progression (grade B, aMMP-8 level $\geq 20 \mathrm{ng} / \mathrm{ml}$ ) and with a rapid rate of progression (grade C, aMMP-8 
level > $30 \mathrm{ng} / \mathrm{ml}$ ) [4]. In another study by Sorsa et al. [14] the DentoAnalyzer-PoC-test and IFMA could comparably detect GCF MMP-8. Using Western Immunoblot it was confirmed that they had the potential to identify activated $55 \mathrm{kDa}$ MMP-8 in periodontitis affected sites. The Amersham ELISA results for total MMP-8 were not in line with the findings. Lateral flow chromatography evaluating neutrophil collagenase-2 (MMP-8) was also tested. PerioMarker was $96 \%$ sensitive for poor oral hygiene, $95 \%$ sensitive for at least two sites with periodontal pockets, and $82.6 \%$ sensitive for at least two sites with bleeding on probing [36].

Some studies pinpointed plausible associations of various periodontal microbiota with levels of MMP-8 in oral fluids. The presence of $T$. denticola and $T$. forsythia was associated with significantly higher concentration of MMP-8 in GCF [37]. Subsequently, T. denticola was shown to produce serine protease (dentilisin) that converted pro-MMP-8 to a-MMP [38, 39]. Gingipain produced by $P$. gingivalis has a similar property [40]. In our study the prevalence of $T$. forsythia was more frequent in periodontitis grade $\mathrm{C}$ as compared to periodontitis grade $\mathrm{B}$, but its occurrence did not correlate with MMP concentrations. aMMP-8 levels evaluated with the previously described PerioMarker test were connected with the prevalence of $P$. gingivalis, $T$. forsythia, $P$. intermedia, Parvimonas micra, Campylobacter rectus and E. nodatum $(p<0.05)$ [41]. Salminen et al. [40] proposed that combined measurement of biomarkers and various pathogens might enhance diagnostic accuracy. The calculated cumulative risk score from three salivary biomarkers (MMP-8, IL-1 $\beta, P$. gingivalis) was more precise in the diagnosis of periodontitis than any marker alone. A recent systematic review stated that the combination of MMP-8 and IL-6 showed the best diagnostic performance [42]. What is more, a combination of MMP-8, MIP- $1 \alpha$, IL- $1 \beta$ and IL- 6 could be useful for distinction between gingivitis and periodontitis as well as for periodontitis compared with gingival health. However, the sophistication and economic burden of such an approach increases significantly. In our study correlations between metalloproteinase levels in GCF and abovementioned microbiota were not found. However, higher concentrations of total and active MMP-8 were linked to $F$. nucleatum presence. $F$. nucleatum is a Gram-negative, nonmotile, anaerobic species, that is part of the "orange complex" [43]. The prevalence of this pathogen increases with the severity of periodontal diseases and the progression of inflammation. Moreover, its presence supports colonization of periodontal pockets by $P$. gingivalis and other microbiota with greater pathogenic potential [44]. F. nucleatum possesses several virulence factors, such as adhesins, outer membrane proteins (Fap2 RadD), endotoxins (LPS), secretion of serine proteases and production of ammonium and butyrate [45]. In gingival epithelial cells, it led to nuclear factor $\kappa \mathrm{B}(\mathrm{NF}-\kappa \mathrm{B})$ activation, leading to enhanced expression of proinflammatory cytokine IL- 8 and IL-1 $\beta$ secretion [46].

There are some limitations to our study that have to be addressed. First, samples were collected only from 24 subjects; thus the study population is relatively small, which limits the expectable statistical power and possibility to draw consistent conclusions. It also implies a chance of type 2 error for some comparisons and represents the biggest shortcoming of this study. Some potential associations might have been overlooked due to reduced power of statistical analysis. Moreover, the cross-sectional examination does not allow sound causative conclusions. The size of the group was determined by financial limitations as the study was self-supported by the authors. Second, the optimal procedure in assessing periodontal grade is observation of patients over several years, but in the present study it was made based on clinical and radiological examination. Patients were divided into grade B and grade $\mathrm{C}$. However, patients with grade A (slow rate of progression) were not included. It would be interesting to see if there were differences between grade A vs. B and A vs. C for total MMP-8 and aMMP-8 tests. Third, the evaluation of selected microbiota was limited by utilization of a commercially available PCR-based test. The analysis of microbiological findings with regards to clinical and periodontal parameters would be of great value, but a following subgroup analysis in the present research would be too small. Evaluation of bacterial species in blood samples should be part of future investigations. It seems rational to carry on with longitudinal studies on a larger number of patients, for example, in a multicenter study. Different periodontal diagnosis and the importance of behavioral factors should also be taken into account. For example, smoking has been established as a modifying risk factor for periodontitis and stratifying results of MMP-8 use as a biomarker in smokers vs. non-smoker patients will provide very interesting findings. Other confounding factors, such as obesity and gender, need to be addressed in detail. Also, comparing the two tests used in this study to other available aMMP-8/ MMP-8 tests might be another potential idea for future projects. There are currently available many different MMP-8 tests and they differ in methods and antibodies, which can have an impact on the results and interpretations of the value of MMP-8 in periodontal diagnostics. Future studies should also evaluate the relationship between MMP-8 and other markers. It would all be beneficial in personal therapeutic intervention and preventive strategies for the occurrence of periodontal diseases.

Both total and active MMP- 8 concentrations reflected periodontal destruction in this report and correlated with the prevalence of $F$. nucleatum. All in all, the aMMP-8 Test was easier and more convenient to handle. Based on the outcomes of our study, we can conclude that MMP-8 evaluation in GCF might prove useful for diagnosis of periodontal diseases. However, due to the relatively small 
sample size these findings should be analyzed with caution. It can be speculated that in future molecular analysis of different inflammatory mediators in a qualitative and quantitative manner will become easier, more convenient and cost-effective. Noninvasive point-of-care testing of aMMP-8, which could support dentists in their responsibility of overseeing patients' oral health, might represent the greatest potential.

\section{The authors declare no conflict of interest.}

\section{References}

1. Birkedal-Hansen H (1993): Role of matrix metalloproteinases in human periodontal diseases. J Periodontol 64: 474-484.

2. Sorsa T, Gieselmann D, Arweiler NB, Herníndez MA (2017): A quantitative point of care test for periodontal and dental peri-implant diseases. Nat Rev Dis Primers 3: 17070.

3. de Morais EF, Pinheiro JC, Leite RB, et al. (2017): Matrix metalloproteinase- 8 levels in periodontal disease patients: a systematic review. J Periodont Res 53: 156-163.

4. Sorsa T, Alassiri S, Grigoriadis A, et al. (2020): Active MMP8 (aMMP- 8$)$ as a grading and staging biomarker in the periodontitis classification. Diagnostics (Basel) 10: pii: E61.

5. Sorsa T, Tjäderhane L, Salo T (2004): Matrix metalloproteinases (MMPs) in oral diseases. Oral Dis 10: 311-318.

6. Sorsa T, Tjäderhane L, Konttinen YT, et al. (2006): Matrix metalloproteinases: contribution to pathogenesis, diagnosis and treatment of periodontal inflammation. Ann Med 38: 306-321.

7. Kiili M, Cox SW, Chen HY, et al. (2002): Collagenase-2 (MMP-8) and collagenase-3 (MMP-13) in adult periodontitis: molecular forms and levels in gingival crevicular fluid and immunolocalisation in gingival tissue. J Clin Periodontol 29: 224-232.

8. Konopka L, Pietrzak A, Brzezińska-Błaszczyk E (2012): Effect of scaling and root planing on interleukin-1 $\beta$, interleukin-8 and MMP-8 levels in gingival crevicular fluid from chronic periodontitis patients. J Periodontal Res 47: 681-688.

9. Ciantar M, Caruana DJ (1998): Periotron 8000: calibration characteristics and reliability. J Periodont Res 33: 259-264.

10. Franco C, Patricia HR, Timo S, et al. (2011): Matrix metalloproteinases as regulators of periodontal inflammation. Int J Mol Sci 18: pii: E440.

11. Al-Majid A, Alassiri S, Rathnayake N, et al. (2018): Matrix metalloproteinase- 8 as an inflammatory and prevention biomarker in periodontal and peri-implant diseases. Int J Dent 2018: 7891322.

12. Zhang L, Li X, Yan H, Huang L (2018) Salivary matrix metalloproteinase (MMP)-8 as a biomarker for periodontitis. A PRISMA-compliant systematic review and meta-analysis. Medicine (Baltimore) 97: e9642.

13. Sorsa T, Ulvi K, Nwhator S, et al. (2016): Analysis of matrix metalloproteinases, especially MMP-8, in GCF, mouthrinse and saliva for monitoring periodontal diseases. Peridontol 2000 70: 142-163.

14. Sorsa T, Herníndez M, Leppilahti J, et al. (2010): Detection of gingival crevicular fluid MMP-8 levels with different laboratory and chair-side methods. Oral Dis 16: 39-45.

15. O'Leary TJ, Drake RB, Naylor JE (1972): The plaque control record. J Periodontol 43: 38-46.
16. Ainamo J, Bay I (1975): Problems and proposal for recording gingivitis and plaque. Int Dent J 25: 229-235.

17. Tonetti MS, Greenwell H, Kornman KS (2018): Staging and grading of periodontitis: Framework and proposal of a new classification and case definition. J Clin Periodontol 45 (Suppl 20): 149-161.

18. Page RC (1991): The role of inflammatory mediators in the pathogenesis of periodontal disease. J Periodontal Res 26 (3 Pt 2): 230-242.

19. Salminen A, Gursoy UK, Paju S, et al. (2014): Oral salivary biomarkers of bacterial burden, inflammatory response, and tissue destruction in periodontitis. J Clin Periodontol 41: 442-450.

20. Gursoy UK, Könönen E, Pradhan-Palikhe P, et al. (2010): Salivary MMP-8, TIMP-1 and ICTP as markers of advanced periodontitis. J Clin Periodontol 37: 487-493.

21. Gursoy UK, Könönen E, Huumonen S, et al. (2013): Salivary type I collagen degradation end-products and related matrix metalloproteinases in periodontitis. J Clin Periodontol 40: $18-25$.

22. Gupta N, Gupta ND, Gupta A, et al. (2015): Role of salivary matrix metalloproteinase-8 (MMP-8) in chronic periodontitis diagnosis. Front Med 9: 72-76.

23. Johnson N, Ebersole JL, Kryscio RJ, et al. (2016): Rapid assessment of salivary MMP-8 and periodontal disease using lateral flow immunoassay. Oral Dis 22: 681-687.

24. Arias-Bujanda N, Regueira-Iglesias A, Balsa-Castro C, et al. (2020): Accuracy of single molecular biomarkers in saliva for the diagnosis of periodontitis: a systematic review and meta-analysis. J Clin Periodontol 47: 2-18.

25. Arias-Bujanda N, Regueira-Iglesias A, Balsa-Castro C, et al. (2019): Accuracy of single molecular biomarkers in gingival crevicular fluid for the diagnosis of periodontitis: a systematic review and meta-analysis. J Clin Periodontol 46: 1166-1182.

26. Kinney JS, Morelli T, Oh M, et al. (2014): Crevicular fluid biomarkers and periodontal disease progression. J Periodontol 41: 113-120.

27. Leppilahti JM, Hernŕndez-Rios PA, Gamonal JA, et al. (2014): Matrix metalloproteinase and myeloperoxidase in GCF provide site-specific diagnostic value for chronic periodontitis. J Clin Periodontol 41: 348-356.

28. Leppilahti JM, Sorsa T, Kallio MA, et al. (2015): The utility of gingival crevicular fluid matrix metalloproteinase- 8 response patterns in prediction of site-level clinical treatment outcome. J Periodontol 86: 777-787.

29. Noack B, Kipping T, Tervahartiala T, et al. (2017): Association between serum and oral matrix metalloproteinase- 8 test in finnish adolescents. J Periodontol 88: 464-472.

30. Leppilahti JM, Ahonen MM, Herń́ndez M, et al. (2011): Oral rinse MMP-8 point-of-care immuno test identifies patients with strong periodontal inflammatory burden. J Periodontol 17: 115-122.

31. Baeza M, Garrido M, Hernńndez-Rios, et al. (2016): Diagnostic accuracy for apical and chronic periodontitis biomarkers in gingival crevicular fluid: an exploratory study. J Clin Periodontol 43: 34-45.

32. Alassiri S, Parnanen P, Rathnayake N, et al. (2018): Ability of quantitative, specific and sensitivity point-of-care/chair side oral fluid immunotests for aMMP-8 to detect periodontal and peri-implant diseases. Dis Markers 2018: 1306396.

33. Räisänen IT, Heikkinen AM, Nwhator SO, et al. (2019): On the diagnostic discrimination ability of mouthrinse and salivary aMMP-8 point-of-care testing regarding perodontal health and disease. Duagn Microbiol Infect Dise 95: 114871. 
34. Izadi Borujeni S, Mayer M, Eickholz P (2015): Activated metalloproteinase-8 in saliva as diagnostic test for periodontal disease? A case-control study. Med Microbiol Immunol 204: 665-672.

35. Räisänen IT, Sorsa T, van der Schoor GJ, et al. (2019): Active metalloproteinase-8 point-of-care ( $\mathrm{PoC}$ )/chairside mouthrinse test vs bleeding on probing in diagnosing subclinical periodontitis in adolescents. Diagnostics 9: 34.

36. Nwhator SO, Ayanbadejo PO, Umeizudike KA, et al. (2014): Clinical correlates of a lateral-flow immunoassay oral risk indicator. J Periodontol 85: 188-194.

37. Yakob M, Kari K, Tervahartiala T, et al. (2012): Associations of periodontal microorganisms with salivary proteins and MMP-8 in gingival crevicular fluid. Clin Periodontol 39: 256-263.

38. Sorsa T, Ingman T, Suomalainen K, et al. (1992): Identification of proteases from periodontopathogenic bacteria as activators of latent human neutrophil and fibroblast-type interstitial collagenases. Infect Immun 60: 4491-4495.

39. Yakob M, Heurman JH, Sorsa T, Söder B (2013): Treponema denticola associates with increased levels of MMP-8 and MMP-9 in gingival crevicular fluid. Oral Dis 19: 694-701.

40. Salminen A, Gursoy UK, Paju S, et al. (2014): Salivary biomarkers of bacterial burden, inflammatory response, and tissue destruction in periodontitis. J Clin Periodontol 41: 442-450.

41. Schmalz G, Hübscher AE, Angermann H, et al. (2019): Associations of chairside salivary aMMP-8 findings with periodontal parameters, potentially periodontal pathogenic bacteria and selected blood parameters in systematically healthy adults. Diagn Microbiol Infect Dis 95: 179-184.

42. Sukriti KC, Wang XZ, Gallagher JE (2019): Diagnostic sensitivity and specificity of host-derived salivary biomarkers in periodontal disease amongst adults: systematic review. J Clin Periodontol 47: 289-308.

43. Socransky SS, Haffajee AD, Smith CC, Kent RL (1998): Microbial complexes in subgingival plaque. J Clin Periodontol 25: 134-144.

44. Ding Q, Tan KS (2016): The danger signal extracellular ATP is an inducer of Fusobacterium nucleatum biofilm dispersal. Front Cell Infect Microbiol 6: 155.

45. Han YW (2015): Fusobacterium nucleatum: a commensal-turned pathogen. Curr Opin Microbiol 23: 141-147.

46. Hung SC, Huang PR, Almeida-da-Silva CLC, et al. (2018): NLRX1 modulates differentially NLPR3 inflammasome activation and $\mathrm{NF \kappa B}$ signaling during Fusobacterium nucleatum infection. Microbes Infect 20: 615-620. 